\title{
An Algorithm on solving Car Sequencing Problem based on ACO
}

\author{
Yaoyao Bi, Chong Gao, Zhengyu Lu and Yonggang Zhang \\ College of Computer Science and Technology \\ Jilin University \\ Changchun, China, 130012
}

\begin{abstract}
Car sequencing problem is a auto-production-scheduling problem that meets certain conditions on the production line of cars.In this paper, we propose an algorithm, ACO-sequencing, on solving car sequencing problem based on ACO, which introduces the two kinds of structure of pheromone. By comparing the results of two kinds of pheromone structure in the role of problem, we combine the two kinds of pheromone to make it better to solve the problem. At the same time, the optimization results are improved step by step by changing the parameters. Experiment shows, our algorithm has certain advantages in solving efficiency.
\end{abstract}

Keywords - Car sequencing problem; Ant Colony; Optimization; pheromone.

\section{INTRODUCTION}

With the rapid development of mechanization and automation on assembly line, more and more manufacturers are concerned about the Parallel efficiency and scheduling problem of the assembly line. European automotive companies rely on a built-to-order production system, instead of a built-to-inventory perspective which is typical of American or Japanese automakers. The built-to-order perspective tends to generate a very high vehicle diversity in assembly plants, that is very different vehicles and a great variety of car sequences from day to day. Therefore, the order of cars assembled on the assembly line is very important. If this problem can be solved, we can not only reduce time of production, but also optimize the process of production and cut down the cost.
The car sequencing problem was first described by Parello etc. in $1986^{[1]}$. This problem involves scheduling cars along an assembly line, in order to install options (e.g.sun-roof, radio or air-conditioning) on them. Each option is installed by a different station, designed to handle at most a certain percentage of the cars passing along the assembly line, and the cars requiring this option must be spaced such that the capacity of every station is never exceeded. The problem has led to a research upsurge after its presentation .

In 2005, the car manufacturer RENAULT had proposed the subject of the ROADEF challenge concerned the car sequencing problem ${ }^{[2]}$, which attracted twenty-seven teams (a record of participation !) coming from all over the world. So far, the method to solve the problem can be approximately divided into exact approaches (mainly including constraint programming, integer programming and Ad-hoc method) and heuristic approaches (mainly including the ant colony algorithm, genetic algorithm and greedy algorithm). In this paper, we propose an algorithm, ACO-sequencing, on solving Car Sequencing Problem based on ACO (Ant Colony Optimization), which introduces the two kinds of structure of pheromone. Comparing the results of two kinds of pheromone structure in the role of problem, we combine the two kinds of pheromone to make it better to solve the problem. At the same time, we improve the optimization results step by step by changing the parameters. Experiment shows, our 
algorithm has certain advantages in solving efficiency.

\section{MODELING OF PROBLEM}

What we consider is a special kind of car sequencing problem proposed by Renault in 2005 which presents two different classes of Constraint problems, which are batch paint constraint and capacity ratio constraint, and here we solve the second class of constraint problems.

\section{A. Definition of problem}

the car sequencing problem is defined by a quadruple $(\mathrm{V}, \mathrm{O}, \mathrm{p}, \mathrm{q}, \mathrm{r})$,

where

- $\mathrm{V}=\left\{v_{1}, . ., v_{n}\right\}$ is the set of vehicles to be produced;

- $\mathrm{O}=\left\{o_{1}, . ., o_{m}\right\}$ is the set of different options;

- $\mathrm{p}: \mathrm{O} \rightarrow \mathrm{IN}$ and $\mathrm{q}: \mathrm{O} \rightarrow \mathrm{IN}$ define the capacity constraint associated with each option $o_{i} \in 0$; this capacity constraint imposes that, for any subsequence of $q_{i}$ consecutive cars on the line, at most $p_{i}$ of them may require $o_{i}$;

- $\mathrm{r}: \mathrm{V} \times \mathrm{O} \rightarrow\{0,1\}$ defines option requirements, e.g. for each vehicle $v_{j} \in \mathrm{V}$ and for each option $o_{i} \in \mathrm{O}$, $r_{j i}=1$ if $o_{i}$ must be installed on $v_{j}$, and $r_{j i}=0$ otherwise.

$B$. Solution of the problem

a sequence of cars: $\pi=<\begin{array}{ccccc}c_{i 1} & c_{i 2}, & \ldots, & c_{i k} & >\end{array}$

Aggregate sequences: $\Pi_{\mathrm{C}}$

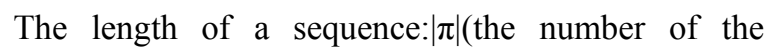
sequence.

The connection of two sequences: $\pi_{1} \subseteq \pi_{2}$.

The cost of a sequence $\pi$ :

$$
\operatorname{cost}(\pi)=\sum_{o_{i} \in O} \sum_{\pi_{k} \subseteq \pi \& \&\left|\pi_{k}\right|=q_{i}} \operatorname{violation}\left(\pi_{k}, o_{i}\right)(1)
$$

Where violation $\left(\pi_{k}, o_{i}\right)=$

$$
\begin{cases}0 \text { if } \sum_{<c i \subseteq \pi k>} \mathrm{r}_{l i} \leq p i \\ 1 & \text { otherwise }\end{cases}
$$

the solution of satisfied data: $\pi=<c_{i 1}$

$c_{i 2}, \quad \cdots c_{i k}>$ to get $\operatorname{cost}(\pi)=0$

the solution of unsatisfied data $: \pi=<c_{i 1}$

$c_{i 2}, \quad \cdots c_{i k}>$ to get the $\operatorname{cost}(\pi)$ as small as possible.

\section{SOLVING ALGORITHM}

With the development of people's research on biological group and social behavior, the algorithm based on artificial-intelligence theory is promoted gradually ${ }^{[3]}$. Ant colony optimization algorithm is an highly innovative heuristic algorithm inspired by the real foraging behavior of ants. When Ant System (AS), which is the first algorithm in line with the ACO framework, is introduced, researchers have been trying to improve the design of it, e.g. Elitist Strategy for Ant System (EAS), Ant-Q algorithm, Ant Colony System (ACS), Max-Min Ant System (MMAS) ${ }^{[4]}$ etc. and they have different effects in practical application. The algorithm proposed in this paper is called ACO-sequencing Algorithm, which is an improved ant algorithm that can be used to solve car sorting problem.

\section{A.Algorithm Framework}

Ant-Solver ${ }^{[5]}$ is an ACO solver for CSPs. The overview of this algorithm is as followed: the ants set value for all variables and when all of them finish assigning values, the pheromone will be updated to complete an iteration.

When one solution that meets the constraints is found or the algorithm reaches the maximal iteration number, the iteration will be stopped.

1 Set parameters and Initialize pheromone trials

2 repeat

3 for $\mathrm{k}$ in 1..nbAnts do: Construct an assignment $A_{k}$

4 Update pheromone trails using $\left\{A_{1}, \ldots, A_{\text {nbAnts }}\right\}$ 
5 until $\operatorname{cost}\left(A_{i}\right)=0$ for some $\mathrm{i} \in\{1 .$. nbAnts $\}$

6 or max cycles reached

\section{B.Solving Thought}

Based on the basic principle of ACO, another three optimization strategies are imported and they are as followed: Elite strategy ${ }^{[6-7]}$ that can accelerate convergence rate, that is to say, only the pheromone between the car nodes that are in the car sequence with the least cost; Maximal-minimal ant system, to prevent the algorithm converging too early, when the pheromone is updated, extreme value is provided to keep it in scope of this maximum number and prevent too early convergence to non-optimal value; Negative feedback, pheromone will volatile to keep the balance of convergence rate, that is to say,pheromone will be reduced by a volatile rate $(\rho)$ before it is updated so that to keep the balance of convergence rate.

According to the analysis of car sorting problem, two information structures ${ }^{[8]}$ are introduced. The cars are abstracted for nodes and the first pheromone is introduced to identify the better cars( to make the car sequence with lowest cost). The pheromone is released among cars by ants to make the choice of next car according to transformation function with lowest expense. For cars with the same requirements belong to the same class,so the second kind of information structure is imported to choose the key cars and the cars with the same type are regarded as one node where the ants will release pheromone.

(1)ACO bases on two pheromones

$$
p\left(c_{i}, \text { cand }, \pi\right)=\frac{\left[\tau_{1}\left(\mathrm{c}_{\mathrm{j}}, \mathrm{c}_{\mathrm{i}}\right)\right]^{\alpha_{1}} \bullet\left[\tau_{2}\left(\operatorname{classOf}\left(\mathrm{c}_{\mathrm{i}}\right)\right)\right]^{\alpha_{2}}}{\sum_{c_{k} \in \text { cand }}\left[\tau_{1}\left(\mathrm{c}_{\mathrm{j}}, \mathrm{c}_{\mathrm{i}}\right)\right]^{\alpha_{1}} \bullet\left[\tau_{2}\left(\operatorname{classOf}\left(\mathrm{c}_{\mathrm{i}}\right)\right)\right]^{\alpha_{2}}}
$$

if the last car of $\pi$ is $c_{j}$

$$
p\left(c_{i}, \text { cand }, \pi\right)=\frac{\left[\tau_{2}\left(\operatorname{classOf}\left(\mathrm{c}_{\mathrm{i}}\right)\right)\right]^{\alpha_{2}}}{\sum_{c_{k} \in \text { cand }}\left[\tau_{2}\left(\operatorname{classOf}\left(c_{k}\right)\right)\right]^{\alpha_{2}}}
$$

if $\pi=<>$ In the above formula,

Cand: Candidate car set $\pi$ : Sequence of chosen cars, $\tau_{Y} C{ }_{j} C\{$ represents the value of first pheromone.
$\tau_{2}\left(\operatorname{classOf}\left(\mathrm{c}_{\mathrm{i}}\right)\right.$ represents the second pheromone in the type that $c_{i}$ belongs to.

(2)ACO-sequencing bases on the improvement in ACO, add environmental variable factor ${ }^{[9]}$.

En is the environmental factor; Ants are affected by the environment in the process of choosing a car, the value is a random number in the interval of $[0,1] . \alpha_{3}$ : The weight of environmental factor.

\section{The Description of ACO-Sequencing Algorithm.}

The description of ACO algorithm solving car sorting problem is as followed: The car sequence is set to 0 initially and when $\pi$ is smaller than the total number, the candidate cars are chosen to make the car with lowest expense and the car is chosen to add to the list $\pi$ to complete a loop. When a car sequence is found or reaches the maximum iteration times, the algorithm stops iteration and output sequence $\pi$.

Input: an instance $(\mathrm{C}, \mathrm{O}, \mathrm{p}, \mathrm{q}, \mathrm{r})$ of the car sequencing problem

a transition probability function $\mathrm{p}: \mathrm{C} \times \mathrm{P}(\mathrm{C})$

$\times \prod_{\mathrm{C}} \rightarrow[0 ; 1]$

Output: a sequence $\pi$ that contains each car of $\mathrm{C}$ once

$1-\pi \leftarrow<>$

2-while $|\pi| \leq|\mathrm{C}|$ do

3 - let $\mathrm{C}-\pi$ denote the set of cars of $\mathrm{C}$ that are not yet sequenced in $\pi$

4- cand $\leftarrow\left\{c_{k} \in \mathrm{G}-\pi \forall \mathrm{c} \mathrm{j} \in \mathrm{C}-\pi, \operatorname{cost}(\pi .<\right.$

$\left.c_{k}>\right) \leq \operatorname{cost}\left(\pi .<c_{j}>\right)$ and

5- $\left.\left(\forall o_{i} \in \mathrm{O}, \quad r_{k i}=r_{j i}\right) \Rightarrow(\mathrm{k} \leq \mathrm{j})\right\}$

6- choose c $\mathrm{i} \in$ cand w.r.t probability $\mathrm{p}\left(c_{i}\right.$, cand, $\pi$ )

$7-\pi \leftarrow \pi \cdot<c_{i}>$

8-end while

9-return $\pi$ 


\section{D.Experimental test}

In order to test the performance of the algorithm in this paper, test data from CSPlib ${ }^{[10]}$ (A problem library for constraints ) is introduced and divided into two groups and one is satisfied data and the other one is unsatisfied data. The size of each group is one hundred, two hundred, three hundred and four hundred according to the size of cars. The experimental environment is Windows XP operating problem, COREi3 2.20 GHz CPU and 2G memory. The settings of parameters are as shown in Table 1. The setting of ACO parameters belongs to the first algorithm and the one of ACO-sequencing belongs to the improved algorithm.

Table1 parameter setting

\begin{tabular}{|c|r|r|r|r|r|r|r|r|r|}
\hline & $\alpha_{1}$ & $\rho_{1}$ & $\tau_{\min 1}$ & $\tau_{\max 1}$ & $\alpha_{2}$ & $\rho_{2}$ & $\tau_{\min 2}$ & en & $\alpha_{3}$ \\
\hline AC0 & 2 & 0.01 & 0.01 & 4 & 6 & 0.03 & 1 & - & - \\
\hline $\begin{array}{c}\text { AC0-sequen } \\
\text { cing }\end{array}$ & 2 & 0.01 & 0.01 & 4 & 6 & 0.03 & 1 & $0-1$ & 2 \\
\hline
\end{tabular}

\section{IV.THE ANALYSIS OF FIGURE}

Figure 1 and figure 2 show the results of test data in the CSP lib (the maximum cycle times $\mathrm{n}=5000$, the number of ants $\mathrm{m}=30$ ). From the figures, when the number of the test data is smaller and the test data can be sat, the method of adding environmental factors to solve the problem, which increases the amount of computation by the introduction of environmental factors but speeds up the speed of convergence to the solution of the problem, obviously optimize the result; when the number of the test data is bigger and the test data cannot be satisfied, the method of adding environmental factors to solve the problem, which increases more computation, the effect of optimization is not obvious. The improved algorithm works in the optimization of the problem overall.

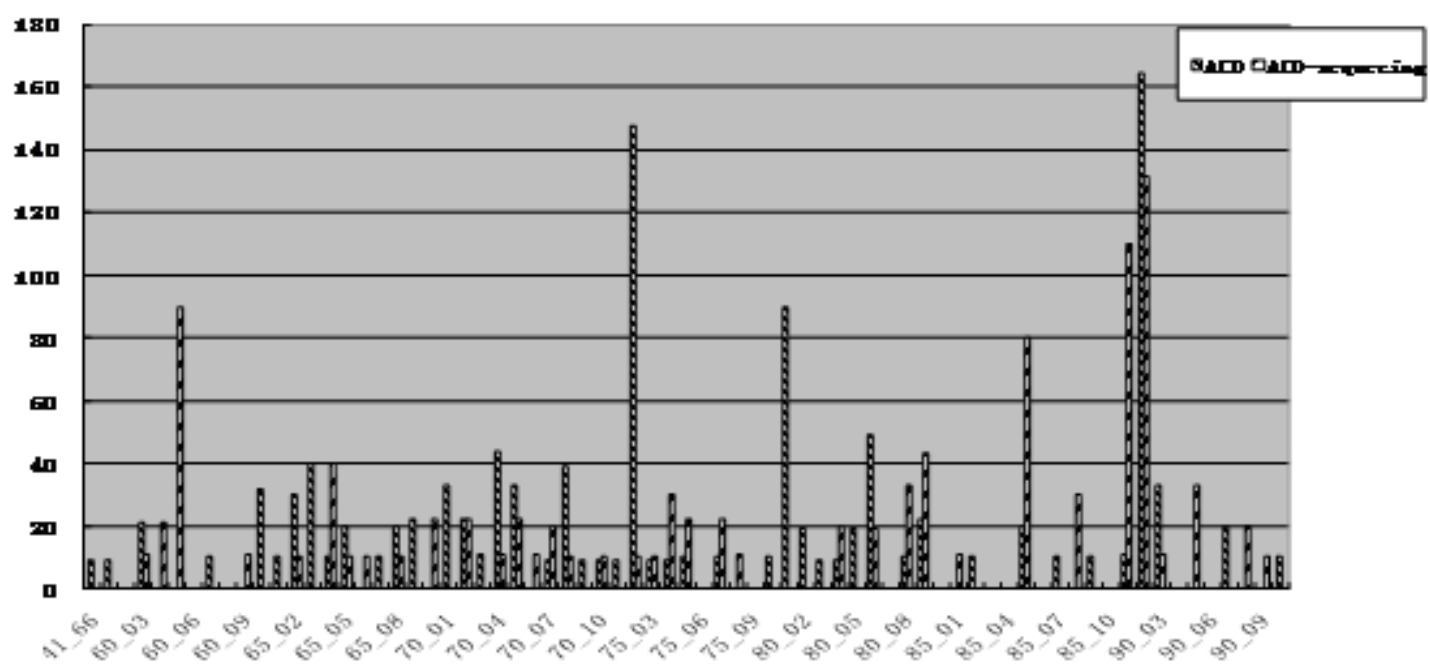

Fig.1 The histogram of the test results of sat data 

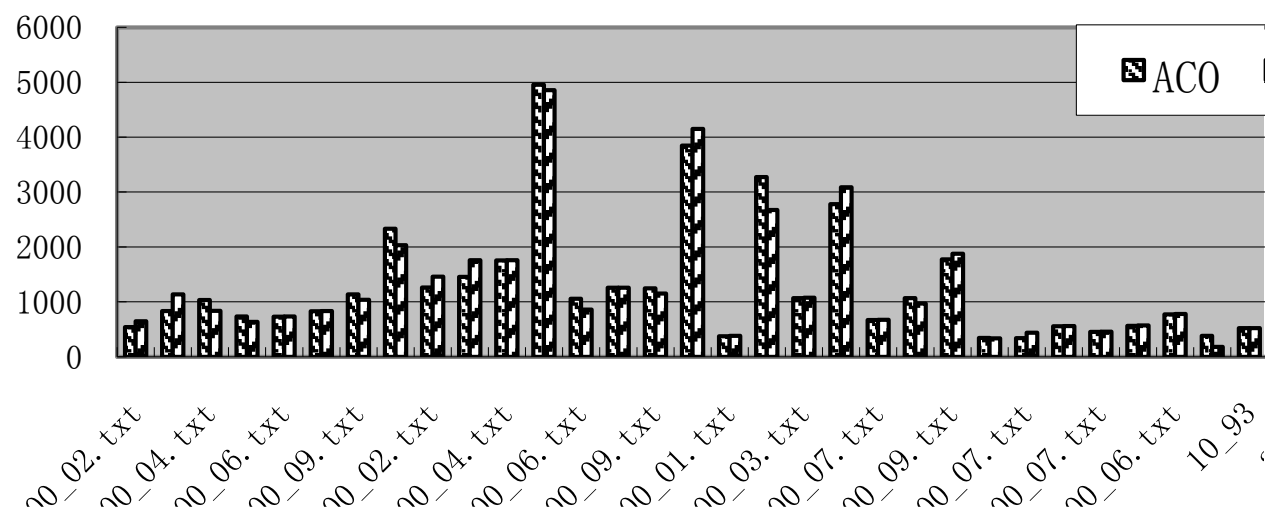

Fig.2 The histogram of the test results of unsatisfied data

\section{CONCLUSION}

In this paper, the car sequencing problem is introduced, extract the effective information based on the analysis and put forward two kinds of pheromone Structure from the problem model. The first kind of pheromone is released between two car nodes. According to this pheromone, we can choose the car nodes to make the sequence cost smaller each time; The second kind of pheromone structure is released on each type of car nodes. According to the two pheromone structure, we propose an algorithm ACO-sequencing, solving the car sequencing problem to choose the key car node that have influences on the cost of sequence greatly. In addition, considering the choice influenced by the environment, we optimize the result by adding the environmental factor. The environment factor is random, so to some extent it has retained its positive effects on the choice .

\section{REFERENCES}

[1] B.D. Parello, W.C. Kabat, and L. Wos. Job-shop scheduling using automated reasoning: a case study of the car sequencing problem. Journal of Automated Reasoning,2:1-42, 1986.
[2] Christine Solnon, Van Dat Cung, Alain Nguyen and Christian Artigues. The car sequencing problem.

[3]Kang qi,Wang lei,An jing.Group intelligence computation.Journal of Shanghai Univer.2004.10, tenth Volume,page 73-76

[4]Zhang jiao-ling.The parameters of the max min ant system.Journal of Guangdong Polytechnic Normal Univer.2007.page 53-56

[5]Xue qiu-shi.An algorithm on constraint solving based Ant colony optimization. jilin university: jilin university,2013

[6] Zhang su-bing,Lv guo-ying,Liu Su-ming.Hierarchical QoS routing based on Ant Algorithm.Journal of circuits and systems. March in 2000,fifth Volume,page 1-5

[7]CAI Xiao-long,ZHANG Xin-min.Research on multiple QoS anycast routing algorithm based on ant colony optimization algorithm. The eleventh session of the Shenyang Academy of Sciences Conference and Chineseautomobile industry cluster development and Cooperation Forum[c].June in 2014.

[8] Christine Solnon. Combining two Pheromone Structures for Solving the Car Sequencing Problem with Ant Colony Optimization. European Journal of Operational Research191(2008)1043-1055

[9]CHEN Yi-zhao,JIANG Lin.Research of parameters of ant colony algorithm. eleventh Volume,page 9080-84

[10]I.P. Gent and T. Walsh. Csplib: a benchmark library for constraints. [Technical report] APES-09-1999 , 1999. available from http://csplib.cs.strath.ac.uk/. A shorter version appears in CP99. 